\title{
Some Results in Einstein's Unified Field Theory
}

\author{
E. G. Straus \\ University of California, Los Angeles, California
}

\section{INTRODUCTION}

$I^{\mathrm{N}}$ $\mathrm{N}$ this paper we discuss some results in the unified field theory proposed by A. Einstein. ${ }^{1}$ We first give a brief summary of that theory:

The fundamental field quantities are the real symmetric tensor density $\mathfrak{g} \frac{i k}{}$, the imaginary tensor density $\mathrm{g}^{i k l}$ which is antisymmetric in each pair of indices, and the Hermitian affine connections $\Gamma_{i k}^{l}=\bar{\Gamma}_{k i}^{l}$.

We define the Hermitian tensor density

$$
\mathfrak{g}^{i k}=\mathfrak{g}^{\underline{i k}}+\mathfrak{g}_{, s}^{i k s},
$$

where the comma stands for ordinary differentiation, and the corresponding Hermitian tensors $g^{i k}$ and $\dot{g}_{i k}$. We define the curvature tensor

$$
R_{k l m}^{i}=\Gamma_{k l, m}^{i}-\Gamma_{k m, l}^{i}-\Gamma^{i}{ }_{s l} \Gamma_{k m}^{s}+\Gamma_{s m}^{i} \Gamma_{k l}^{s},
$$

which has the Hermitized contraction

$$
\begin{aligned}
R_{i k}=\Gamma_{i k, a}^{a}-\frac{1}{2}\left(\Gamma_{i a, k}^{a}+\Gamma_{a k, i}^{a}\right) & \\
& -\Gamma^{a}{ }_{i b} \Gamma_{a k}{ }^{b}+\frac{1}{2} \Gamma^{a}{ }_{i k}\left(\Gamma^{b}{ }_{a b}+\Gamma_{b a}^{b}\right) .
\end{aligned}
$$

We then define the Hamiltonian function

$$
\mathfrak{h}=\mathfrak{g}^{i k} R_{i k}
$$

and we obtain the field equations from the variational principle

$$
\delta \int \mathfrak{h} d V=0
$$

The resulting equations are

$$
\begin{gathered}
\mathfrak{g}^{i k}{ }_{i l}=\mathfrak{g}^{i k}, l+\mathfrak{g}^{s k} \Gamma_{. s l}^{i}+\mathfrak{g}^{i s} \Gamma_{l s}^{k}-\frac{1}{2} \mathfrak{g}^{i k}\left(\Gamma^{s} l s+\Gamma_{s l}^{s}\right)=0, \\
R_{\underline{i k}}=0, \\
R_{i k, l}+R_{k l, i}+R_{l i, k}=0 .{ }^{2}
\end{gathered}
$$

The first system of equations is equivalent to either of the systems

$$
\begin{aligned}
& g^{i k}{ }_{; l}=g^{i k}, l+g^{s k} \Gamma_{s l}^{i}+g^{i s} \Gamma^{k}{ }_{l s}=0, \\
& g_{i k ; l}=g_{i k, l}-g_{s k} \Gamma^{s}{ }_{i l}-g_{i s} \Gamma^{s}{ }_{l k}=0 .
\end{aligned}
$$

The relation $\mathfrak{g}_{v}^{i k}=\mathfrak{g}^{i k s}, s$ is equivalent to the equations

$$
\mathrm{g}^{i k}, k=0 \text {, }
$$

or

$$
\Gamma_{i s}^{s}=0 .
$$

Our first section deals with the solution of the equations $g_{i k ; l}=0$ in terms of the $\Gamma$. This is a far more difficult problem than the experience with Riemannian geometry would lead us to believe.

1 A. Einstein, Ann. Math. 46, 578 (1945); A. Einstein and E. G. Straus, Ann. Math. 47, 731 (1946); A. Einstein, Rev. Mod. Phys. 20, 35 (1948).

2 The symbols - and $v$ are used to indicate symmetry and antisymmetry in these indices.
The second section gives a short discussion of the stronger equations $R_{k l m}^{i}=0$ which in this non-Riemannian geometry do not characterize flat space.

The rest of the paper is devoted to the problem of the existence of regular solutions of the field equations. Indeed there is good evidence to show that the field equations are not sufficiently restrictive so that not all their solutions have physical significance. If the field equations are to be augmented by a condition in the large then the condition of the regularity of the field quantities seems to be the most natural.

In our discussion space is assumed to be the topological equivalent of four dimensional Euclidean space. In the third section we obtain a certain negative result whose physical meaning might be stated as follows: "a particle with mass cannot be represented by a static solution which is everywhere regular."

Since it is thus shown that the regular static solutions cannot represent mass the question arises whether there might exist elementary static mass free solutions which could be used as the components of more complex nonstatic solutions which represent mass. The simplest possibility here is that of central symmetry which is discussed in section IV. The result is again negative: There exist no non-trivial regular static centrally symmetric solutions which are asymptotically flat.

This work was done while I was assistant to Professor A. Einstein whose ideas are used at practically every step. My thanks are also due to Professor F. J. Murray who was my advisor and to my wife who helped in checking the calculations.

\section{THE EQUATIONS $g_{i k ; l}=0$}

In a previous paper ${ }^{3}$ we established the fact that the system of equations

$$
g_{i k ; l}=g_{i k, l}-g_{s k} \Gamma_{i l}^{s}-g_{i s} \Gamma^{s} l k=0
$$

cannot be solved for the $\Gamma$ by the same simple trick through which it is solved in the case of symmetric $g_{i k}$. We saw this by studying the determinant of the system (1.1) which cannot be expressed as a power of the determinant $\left|g_{i k}\right|$.

It is now our intention to show how the Eqs. (1.1) can be solved in a comparatively simple manner. For this purpose we start as in the case of symmetric $g_{i k}$ by writing the three equations

$$
\begin{aligned}
& g_{k i, l}=g_{s i} \Gamma^{s}{ }_{k l}+g_{k s} \Gamma^{s}{ }_{l i} \\
& g_{l k, i}=g_{s k} \Gamma^{s}{ }_{l i}+g_{l s} \Gamma^{s}{ }_{i k} \\
& g_{i l, k}=g_{s l} \Gamma^{s}{ }_{i k}+g_{i s} \Gamma^{s}{ }_{k l},
\end{aligned}
$$

\footnotetext{
${ }^{3}$ A. Einstein and E. G. Straus, Ann. Math. 47, 731 (1946).
} 
corresponding to the cyclic permutations of the indices $k, i$. We now multiply the first equation by $-\frac{1}{2} g^{i^{\prime} i} g^{k k^{\prime}}$, the second by $\frac{1}{2} g^{i^{\prime} i} g^{k^{\prime} k}$, and the third by $\frac{1}{2} g^{i i^{\prime}} g^{k k^{\prime}}$. By adding these three equations we obtain:

$$
K^{i^{\prime} k^{\prime}}{ }_{l^{\prime}}=\left(g^{i^{\prime} i} g^{k^{\prime} k} g_{l^{\prime} l}+g^{i i^{\prime}} g^{k k^{\prime}} g_{l l^{\prime}}\right) \Gamma_{i k}^{l},
$$

where

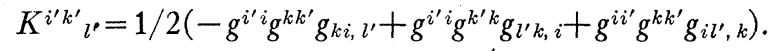

If the $g_{i k}$ were symmetric then the coefficient tensor,

$$
U^{i^{\prime} i k^{\prime} k} k_{l^{\prime} l}=g^{i^{\prime}{ }^{\prime}} g^{k^{\prime} k} g_{l^{\prime} l}+g^{i i^{\prime}} g^{k k^{\prime}} g_{l l^{\prime}}
$$

would equal $2 g^{i^{i} i} g^{k^{\prime} k} g_{l^{\prime} l}$ and would have the trivial inverse:

$$
V_{i^{\prime} i k^{\prime} k^{l^{\prime}} l}{ }^{\prime}=\frac{1}{2} g_{i^{\prime}} g_{k^{\prime} k} g^{l^{\prime} l} .
$$

We remark that the tensor $U$ is real and hence the system (1.3) decomposes into two systems of linear equations, one of 24 equations for the 24 imaginary parts of the $\Gamma$, and one of 40 equations for the 40 real parts of the $\Gamma$. We could proceed to solve the 24 equations for the antisymmetric parts of the $\Gamma$ and once we had solved them we could obtain the symmetric parts of the $\Gamma$ from the equations (real parts of $g_{i k ; l}=0$ ):

$$
g_{\underline{i k}, l}-g_{s k} \Gamma^{s}{ }_{i l}-g_{i s} \Gamma^{s}{ }_{l k}-g_{\underline{s} \underline{k}} \Gamma^{s} \underline{i \underline{i n}}-g_{\underline{i s}} \Gamma^{s} \underline{\underline{k}}=0,
$$

where the coefficients of the symmetric $\Gamma$ are the symmetric parts of the $g$ so that the system of equations can be solved in the customary manner. It is thus possible to reduce the system of 64 equations in 64 unknowns to one system of 24 equations in 24 unknowns and further systems of 4 equations in 4 unknowns. This is the greatest reduction practicable in this case.

However, we shall proceed in a different fashion. We state without proof the following:

Lemma: Given five covariant tensors of rank two, $T_{i k}{ }^{(\alpha)}(\alpha=1, \cdots, 5)$ which are formed from the $g_{i k}$ through purely rational operations then there exists a linear relation

$$
\sum_{\alpha=1}^{5} I^{(\alpha)} T_{i k}(\alpha)=0,
$$

where the $I^{(\alpha)}$ are invariant scalar point functions not all identically equal to zero.

An analogous lemma holds for contravariant and mixed tensors of rank two.

Now, in order to find the inverse of the coefficient tensor $U^{i^{\prime} i k^{\prime} k} k_{l^{\prime} l}$ of (1.3), we determine the systems of tensors $\underset{\alpha}{g_{i k}} \underset{\beta}{g_{i}{ }^{k}} \underset{\gamma}{g^{i k}}$ by the following relations:

$$
\begin{aligned}
& \underset{1}{g_{i k}}=g_{i k}, \underset{0}{g_{i}{ }^{k}}=\delta_{i}{ }^{k}, \underset{1}{g^{i k}}=g^{i k} ;
\end{aligned}
$$

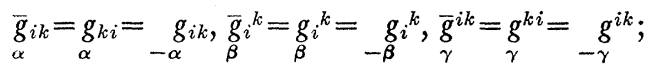

$$
\begin{aligned}
& \underset{\alpha}{g_{s k} g_{i}{ }^{s}}=\underset{\alpha+\beta}{g_{i k}}, \underset{\alpha}{g}, g_{\gamma} g^{s k}=\underset{\alpha+\gamma}{g_{i} k}, g_{\beta} s_{\gamma}^{k} g^{i s}=\underset{\beta+\gamma}{g^{i k}} .
\end{aligned}
$$

The tensors $g_{i k}, g_{i k}, g_{i k}, g_{i k}$ are linearly independent and hence according to the lemma they form a basis for $g_{\alpha}$. In the same manner the tensors ${\underset{-2}{2}}_{i}{ }^{k}, \underset{0}{g_{i}{ }^{k}}, \underset{2}{g_{i}{ }^{k}}, \underset{4}{g_{i}{ }^{k}}$ and $g_{-3}^{i k}, \underset{-1}{g^{i k}}, g_{1}^{i k}, g_{3}^{i k}$, respectively, form a basis for the $\underset{\beta}{g_{i}{ }^{k}}$ and the $g_{\gamma}^{i k}$.

For our purpose we need only the identity between the tensors $g_{-4}{ }^{k}, g_{-2}{ }^{k}, g_{i}{ }^{k}, g_{4}{ }^{k}$. If we denote the two (independent) scalars $g_{s}{ }^{s}$ and $g_{4}{ }^{s}$ by $I_{2}$ and $I_{4}$, respectively, then we obtain

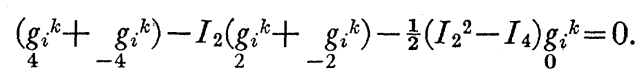

We introduce the abbreviation $I_{0}=\frac{1}{2}\left(I_{2}{ }^{2}-I_{4}\right)$ so that we have

$$
g_{4} i^{k}+\underset{-4}{g_{i}{ }^{k}}=I_{2}\left(g_{i}{ }^{k}+\underset{-2}{\left.g_{i}{ }^{k}\right)}+I_{0} g_{i}{ }^{k} .\right.
$$

We are now in a position to give a formula for the inverse of the tensor $U_{l^{\prime}} l^{i^{i} k^{\prime} k}$. We write:

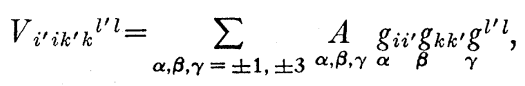

where the coefficients $\underset{\alpha, \beta, \gamma}{A}$ are scalar invariants (functions of $I_{0}, I_{2}$ ) which are to be determined from the relation

$$
U^{i^{\prime} i k^{\prime} k} l_{l^{\prime} l} V_{i^{\prime \prime} i k^{\prime \prime} k^{\prime \prime} l}=\delta_{i^{\prime \prime}} i^{\prime} \delta_{k^{\prime}} k^{\prime} \delta_{l^{\prime}}{ }^{\prime \prime \prime},
$$

or more explicitly

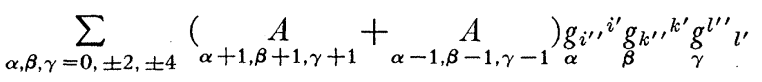

$$
\begin{aligned}
& =\delta_{i^{\prime \prime}}, i^{\prime} \delta_{k^{\prime}}, k^{\prime} \delta_{l^{\prime \prime}},^{\prime} \text {, }
\end{aligned}
$$

with the convention that all $A$ with an index \pm 5 vanish. We can now use the identity (1.6) in order to replace $\underset{-4}{g}$ by a linear combination of $\begin{array}{r}g, g \\ -2\end{array}$ pare the coefficients of the different

$$
g_{\alpha} i^{\prime \prime} i^{\prime} g_{k^{\prime}} k_{\gamma}^{\prime} g_{\gamma}^{l^{\prime \prime}{ }^{\prime}} \quad \alpha, \beta, \gamma=0, \pm 2,4,
$$

we obtain 64 equations for the 64 coefficients $\underset{\alpha, \beta, \gamma}{A}$. We see some properties of the $A$ immediately:

(1) The $A$ are real since the coefficients of the linear equations are real polynomials in the real scalars $I_{0}, I_{2}$.

(2) Since $U$ is real $V$ is real and hence $A=\underset{\alpha, \beta, \gamma}{A}$.

(3) Since $U$ is symmetric in $g^{i^{\prime} i}, g^{k^{\prime \prime} k}, g_{l^{\prime} l}^{\alpha, \beta,}$, we have $V$ symmetric in $g_{i^{\prime}}, g_{k^{\prime} k}, g^{l^{\prime} l}$ and hence $A$ is symmetric in the indices $\alpha, \beta, \gamma$.

Thus the 64 variables reduce to the 10 variables

$$
\begin{array}{ccccc}
\underset{3,3,3}{A}, \underset{3,3,1}{A}, \quad \underset{3,3,-1}{A}, \underset{3,3,-3}{A}, \underset{3,1,1}{A}, \\
\underset{3,1,-1}{A}, \underset{3,1,-3}{A}, \quad \underset{1,1,1}{A}, \underset{1,1,-1}{A}, \underset{1,1,-3}{A} .
\end{array}
$$


The determinant of the system of equations for these $A$ is

$$
\begin{aligned}
\left(1+I_{2}-\frac{1}{2} I_{0}\right)^{2}\left(1+I_{0}+I_{2}\right)\left[I_{0}{ }^{2}+\right. & \left(-1+3 I_{2}\right) I_{0} \\
+ & \left.\left(-2+4 I_{2}+2 I_{2}{ }^{2}+I_{2}{ }^{3}\right)\right]
\end{aligned}
$$

and the $A$ turn out to be rather complicated rational expressions in the $I_{0}, I_{2}$.

The system $g_{i k ; l}=0$ then has the solution

$$
\sum_{\alpha, \beta, \gamma} \underset{\alpha, \beta, \gamma}{A} g_{i^{\prime} i} g_{k^{\prime}} k g^{g^{\prime} l} K^{i^{\prime} k^{\prime}{ }^{\prime}} .
$$

Aside from the unwieldiness of the scalars $A$ this solution has the unpleasant feature that one of the factors of the denominator, namely $1+I_{0}+I_{2}$, may vanish without causing the system of equations to become singular. This is due to the fact that while the $g_{\alpha}{ }^{k}(\alpha=0, \pm 2,4)$ formed a basis for the mixed tensors of rank two which were constructed from the $g_{i k}$ by rational operations, it is no longer true that the tensors $g_{\alpha}{ }_{\alpha}^{k} g_{\beta}{ }^{m}, g_{\alpha}{ }^{m} g_{\beta} g^{k}(\alpha, \beta=0, \pm 2,4)$ form a basis for the mixed tensors of rank four which are formed from the $g_{i k}$ by rational operations. The latter tensors are linearly dependent.

By using the linear dependences among the tensors $\underset{\alpha}{g_{\alpha}^{i k} g_{l}{ }^{m}}$ and $\underset{\alpha}{g_{i}{ }^{m} g_{\beta}{ }^{k}}$ we can transform our solution (1.9) so that the denominator $1+I_{0}+I_{2}$ is cancelled.

It seems likely that our solution (1.9) cannot be simplified much further. However, there may be a more suitable choice of the basis for the tensors of rank two and the linear dependences among the tensors of rank 4 and 6 may be applicable for a systematic simplification.

\section{THE CASE $\boldsymbol{R}^{i}{ }_{k l m}=0$}

In Riemannian geometry the equation $R^{i}{ }_{k l m}=0$ characterizes flat space, but in our present geometry where the affine connections are non-symmetric, it characterizes the existence of so-called "distant parallelism," that is the existence of four independent vectors $a^{\alpha}{ }_{i}$ such that

$$
a^{\alpha}{ }_{i, k}=\Gamma^{s}{ }_{i k} a^{\alpha}{ }_{s} \quad \alpha=1, \cdots, 4
$$

if we define $a_{\alpha}{ }^{s}$ by the relation

then we obtain

$$
a^{\alpha} a_{\alpha}{ }^{t}=\delta_{s}{ }^{t}
$$

Since we have

$$
\Gamma_{i k}^{l}=a_{\alpha}^{l} a^{\alpha}{ }_{i, k} .
$$

$$
\left(g_{+i k ; l}\right), m-\underset{+-}{\left(g_{i k ; m}\right), l}=-g_{s k} R^{s}{ }_{i l m}-g_{i s} \bar{R}_{k l m}{ }_{k l m}=0,
$$

we know that the field equations $g_{i k ; l}=0$ are compatible with any system of $\Gamma$ which satisfy the equations $R^{i}{ }_{k l m}=0$.

The system of vectors $a^{\alpha}{ }_{i}$ is therefore subject only to two sets of restrictions, one due to the Hermitian character of the $\Gamma$, and one due to the field equations
$\Gamma_{i s}^{s}=0$ :

$$
\begin{gathered}
a_{\alpha}{ }^{l} a^{\alpha}{ }_{i, k}=\bar{a}_{\alpha}{ }^{l} \bar{a}_{k, i}^{\alpha} \\
a_{\alpha}{ }^{s}\left(a^{\alpha}{ }_{i, s}-a^{\alpha}{ }_{s, i}\right)=0 .
\end{gathered}
$$

While it seems difficult to find the general solution of this overdetermined system of equations, we can get an idea of the manifold of solutions by a parametric expansion. We write

$$
a^{\alpha}{ }_{i}=\underset{0}{a} a_{i}+\underset{1}{a^{\alpha}}{ }_{i}+\underset{2}{a^{\alpha}}+\cdots .
$$

Without loss of generality we can choose $\underset{0}{a^{\alpha}}{ }_{i}=\delta^{\alpha}{ }_{i}$. The first order term of the first system of (2.2) then becomes:

$$
a_{1}^{l}{ }_{i, k}=\bar{a}_{1}^{l}{ }_{k, i}
$$

or if we set $\underset{1}{a}=\underset{1}{b}+i c$ :

$$
\begin{aligned}
& b_{1, k}^{l}=b_{1}^{b_{k, i}} \\
& c_{1}^{l}{ }_{i, k}+c_{1}^{c_{k, i}}=0,
\end{aligned}
$$

which yields the solution

$$
\begin{aligned}
& b_{1}^{l_{i}}=b^{l}, i \\
& c_{1}^{l}=A^{l}{ }_{i s} x_{s} \text { with } A^{l_{i k}}+A^{l_{k i}}=0 .
\end{aligned}
$$

The first order term of the second system of (2.2) then yields

$$
A_{i s}^{s}=0 .
$$

By a suitable choice of the coordinates we can make $b_{i} l_{i}=0$. Thus the system is completely determined through the choice of the constants $A_{i k}$. The number of independent constants is four.

\section{ON THE NON-EXISTENCE OF REGULAR STATIONARY SOLUTIONS REPRE- SENTING MASS ${ }^{4}$}

Einstein and Pauli ${ }^{5}$ showed that no everywhere regular static solution of the field equations of the general theory of relativity for which space is asymptotically flat $\left(g_{i k} \rightarrow \eta_{i k}\right.$ as $\left.r\left[=\left(x_{1}{ }^{2}+x_{2}{ }^{2}+x_{3}^{2}\right)^{\frac{1}{2}}\right] \rightarrow \infty\right)$. represents mass; that is to say $g^{i k}-\eta^{i k}=o(1 / r)$. Their proof was based on the construction of a surface integral relation for the field quantities.

When the field equations are obtained from a relativistically invariant variational principle,

$$
\delta \int_{V} \mathfrak{h} d V=0, \quad V=\text { four dimensional region, }
$$

${ }^{4}$ For a different proof of the result of this section see Papapetron, Phys. Rev. 73, 1105 (1948).

${ }^{5} \mathrm{~A}$. Einstein and W. Pauli, "On the non-existence of regular stationary solutions of relativistic field equations," Ann. Math. 44, 131 (1943). 
then an infinitesimal change of the coordinate system

$$
x_{i}^{*}=x_{i}+\xi_{i}, \quad x^{i *}=x^{i}-\xi^{i}
$$

produces new field quantities and a new scalar density $\mathfrak{h}^{*}$ so that the variational principle remains satisfied. If we call the increments $\delta \mathfrak{h}, \delta \mathfrak{g}^{i k}, \delta \Gamma^{l}{ }_{i k}$ etc., then we obtain (if we let $\mathfrak{h}=\mathfrak{h}\left(\mathfrak{g}^{\underline{i k}}, \mathfrak{g}^{i k s}, \Gamma_{i k}^{l}, \Gamma_{i k, m}^{l}\right)$ ):

$$
\begin{aligned}
0=\int_{V} \delta \mathfrak{h} d V= & \int_{V}\left[\frac{\partial \mathfrak{h}}{\partial \mathfrak{g} \underline{i k}} \delta \mathfrak{g}^{i \underline{k}}+\frac{\partial \mathfrak{h}}{\partial \mathfrak{g}_{v}^{i k}} \delta \mathfrak{g}^{i k}\right. \\
& \left.+\frac{\partial \mathfrak{h}}{\partial \Gamma^{l}{ }_{i k}} \delta \Gamma^{l}{ }_{i k}+\frac{\partial \mathfrak{h}}{\partial \Gamma^{l}{ }_{i k, m}} \delta \Gamma^{l}{ }_{i k, m}\right] d V .
\end{aligned}
$$

But we have

$$
\delta \mathfrak{h}=-\left(\mathfrak{h} \xi^{s}\right), s,
$$

and due to the field equation $R_{\underline{i k}}=0$,

also

$$
\delta \mathfrak{h}=-\left(R_{i k} \mathfrak{g}^{i k} \xi^{s}\right)_{, s} ;
$$

$$
\delta \mathfrak{g}^{i k}=\mathfrak{g}^{s k} \xi_{, s}^{i}+\mathfrak{g}^{i s} \xi^{k}, s-\left(\mathfrak{g}^{i k} \xi^{s}\right), s
$$

and hence

$$
\begin{aligned}
R_{i k} \delta \mathfrak{g}^{i k} & =2 R_{i k} \mathfrak{g}^{s k} \xi_{, s}^{i}-\left(R_{i k} \mathfrak{g}^{i k} \xi^{s}\right)_{, s}+\mathfrak{g}^{i k} R_{i k, s} \xi^{s} \\
& =\delta \mathfrak{h}+2 R_{i k} \mathfrak{g}^{s k} \xi_{, s}^{i}+\mathfrak{g}^{i k} R_{i k, s} \xi^{s} .
\end{aligned}
$$

Because of the field equations $R_{i k, s}+R_{k s, i}+R_{s i, k}=0$ and $\mathfrak{g}_{v}^{s k}, s=0$ this becomes

$$
\begin{aligned}
R_{i k} \delta \mathfrak{g}_{v}^{i k} & =\delta \mathfrak{h}+2 R_{i k} \mathfrak{g}^{s k} \xi_{, s}^{i}-\mathfrak{g}_{v}^{i k}\left(R_{k s, i}+R_{s i, k}\right) \xi^{s} \\
& =\delta \mathfrak{h}+2 R_{i k} \mathfrak{g}_{v}^{s k} \xi_{, s}^{i}+2 \mathfrak{g}_{v}^{s k} R_{i k, s} \xi^{i} \\
& =\delta \mathfrak{h}+2 \mathfrak{g}_{v}^{s k}\left(R_{i k} \xi^{i}\right)_{, s}=\delta \mathfrak{h}+2\left(\mathfrak{g}_{v}^{s k} R_{i k} \xi^{i}\right)_{, s} .
\end{aligned}
$$

Hence (3.1) becomes

$$
\begin{aligned}
\int_{V}\left[\frac{\partial \mathfrak{h}}{\partial \mathfrak{g} \underline{i k}} \delta \mathfrak{g}^{i k}+2\left(\mathfrak{g}^{s k} R_{i k} \xi^{i}\right)_{s}\right. \\
\left.+\left\{\frac{\partial \mathfrak{h}}{\partial \Gamma^{l}{ }_{i k}}-\left(\frac{\partial \mathfrak{h}}{\partial \Gamma^{l}{ }_{i k, s}}\right)_{, s}\right\} \delta \Gamma^{l}{ }_{i k}\right] d V \\
+\int_{S} \frac{\partial \mathfrak{h}}{\partial \Gamma^{l}{ }_{i k, s}} \delta \Gamma^{l}{ }_{i k} n_{s} d S=0
\end{aligned}
$$

where $S$ is the (three dimensional) surface of $V$ and $n_{i}$ is the $i$-component of the unit normal vector to $S$. On the right side of (3.2) we have the variations of $\mathfrak{h}$ with respect to $\mathfrak{g}^{\underline{i k}}$ and $\Gamma_{i k}^{l}$, which vanish due to the field equations. Thus (3.2) becomes

$$
\int_{V} 2\left(\mathfrak{g}^{s k} R_{i k} \xi^{i}\right)_{, s} d V+\int_{S} \frac{\partial \mathfrak{h}}{\partial \Gamma_{i k, s}^{l}} \delta \Gamma^{l}{ }_{i k} n_{s} d S=0,
$$

or

$$
\int_{S}\left[2 \mathfrak{g}^{s k} R_{i k} \xi^{i}+\frac{\partial \mathfrak{h}}{\partial \Gamma^{l}{ }_{i k, s}} \delta \Gamma^{l}{ }_{i k}\right] n_{s} d S=0
$$

We have

$$
\frac{\partial \mathfrak{h}}{\partial \Gamma_{i k, s}^{l}}=\mathfrak{g}^{i k} \delta_{l}{ }^{s}-\frac{1}{2} \mathfrak{g}^{i s} \delta_{l}{ }^{k}-\frac{1}{2} \mathfrak{g}^{s k} \delta_{l}{ }^{i}
$$

and hence (3.3) becomes

$$
\begin{aligned}
& \int_{S}\left[2 \mathfrak{g}^{s k} R_{i k} \xi^{i}+\mathfrak{g}^{i k}\left(\delta \Gamma^{s}{ }_{i k}\right.\right. \\
&\left.\left.\quad-\frac{1}{2} \delta_{k}{ }^{s} \delta \Gamma^{t}{ }_{i t}-\frac{1}{2} \delta_{i}{ }^{s} \delta \Gamma^{t}{ }_{t i}\right)\right] n_{s} d S=0 .
\end{aligned}
$$

In general there seems to be little that can be achieved with the help of this relation. However, if all the field quantities are assumed to be static, that is independent of $x_{4}=t$, then we can choose $V$ to be a right cylinder with the bases $x_{4}=t_{1}, x_{4}=t_{2}$ and $\xi^{i}$ independent of $x_{4}$ for $i=1,2,3$ with $\xi^{4}=c x_{4}$. Now if we let $B_{1}, B_{2}$ be the bases and $L$ the lateral surface of our cylinder then in the integration over $S$ the integrals over $B_{1}$ and $B_{2}$ cancel except for the term

$$
\int_{B} 2 \mathfrak{g}^{4 k} R_{4 k} c\left(t_{2}-t_{1}\right) d B=2 c\left(t_{2}-t_{1}\right) \int_{B} \mathfrak{g}^{4 k s},{ }_{,} R_{4} k d B
$$

but, since we have $R_{4 k, s}-R_{4 s, k}=0$, we obtain

$$
\begin{aligned}
2 c\left(t_{2}-t_{1}\right) \int_{B}\left(\mathfrak{g}^{4 k s} R_{4 k}\right), s & \\
& =2 c\left(t_{2}-t_{1}\right) \int_{C} \mathfrak{g}^{4 k s} R_{4 k} n_{s} d C,
\end{aligned}
$$

where $C$ is the (two dimensional) boundary of $B$ and $n_{\varepsilon}$ is the $s$-component of the unit normal vector to $C$ in the plane of $B$.

The integral of $2 \mathfrak{g}^{s k} R_{4 k} \xi^{4} n_{s}$ over $L$ yields

$$
2 c \int_{t_{1}}^{t_{2}} x_{4} d x_{4} \int_{C} \mathfrak{g}^{s k} R_{4 k} n_{s} d C=c\left(t_{2}{ }^{2}-t_{1}^{2}\right) \int_{C} \mathfrak{g}^{s k} R_{4 k} n_{s} d C .
$$

Since the remaining integrand is independent of $x_{4}$, it yields

$$
c\left(t_{2}-t_{1}\right) \int_{C} g^{i k}\left(\delta \Gamma^{s}{ }_{i k}-\frac{1}{2} \delta_{k}^{s} \delta \Gamma^{t}{ }_{i t}-\frac{1}{2} \delta_{i}{ }^{s} \delta \Gamma^{t}{ }_{t i}\right) n_{s} d C .
$$

Hence (3.5) becomes

$$
\begin{aligned}
& \int_{C}\left[c\left(t_{2}+t_{1}\right) \mathfrak{g}^{s k} R_{4 k}+2 c \mathfrak{g}^{4 k s} R_{4 k}\right. \\
& \left.\quad+\mathfrak{g}^{i k}\left(\delta \Gamma^{s}{ }_{i k}-\frac{1}{2} \delta_{k}{ }^{s} \delta \Gamma^{t}{ }_{i t}-\frac{1}{2} \delta_{i}{ }^{s} \delta \Gamma^{t}{ }_{t i}\right)\right] n_{s} d C=0 .
\end{aligned}
$$

If we now choose $\xi^{i}=0, i=1,2,3$, then the surface integral relation becomes

$$
\begin{aligned}
0=c \int_{C}\left[\left(t_{2}+t_{1}\right) \mathfrak{g}^{s k} R^{k k}+\right. & 2 \mathfrak{g}^{4 k s} R_{4 k} \\
+ & +\mathfrak{g}^{i k}\left(\Gamma^{4}{ }_{i k} \delta_{4}{ }^{s}-\Gamma_{4 k}{ }^{s} \delta_{i}{ }^{4}-\Gamma^{s}{ }_{i 4} \delta_{k}{ }^{4}\right. \\
& \left.\left.+\frac{1}{2} \delta_{k}{ }^{s} \delta_{i}{ }^{4} \Gamma^{t}{ }_{4 t}+\frac{1}{2} \delta_{i}{ }^{s} \delta_{k}{ }^{4} \Gamma^{t}{ }_{t 4}\right)\right] n_{s} d C
\end{aligned}
$$


and since we have $\Gamma_{4 s}^{s}=\Gamma_{s 4}^{s}=0$ and $n_{4}=0$ :

$\int_{C}\left[\left(t_{2}+t_{1}\right) \mathfrak{g}_{v i k}^{s k} R_{4 k}+2 \mathfrak{g}^{4 k s} R_{4 k}-\mathfrak{g}^{4 k} \Gamma_{4 k}^{s}-\mathfrak{g}^{k 4} \Gamma_{k 4}^{s}\right] n_{\delta} d C=0$.

Since the choice of $t_{1}$ and $t_{2}$ was arbitrary, we obtain

$$
\int_{C}\left(2 \mathfrak{g}^{4 k s} R_{4 k}-\mathfrak{g}^{4 k} \Gamma^{s}{ }_{4 k}-\mathfrak{g}^{k 4} \Gamma^{s}{ }_{k 4}\right) n_{s} d C=0 .
$$

Now under the assumption $\mathrm{g}^{i k} \rightarrow \eta^{i k}$ as $r \rightarrow \infty$, we can write $\mathfrak{g}^{i k}$ in the asymptotic expansion

$$
\mathfrak{g}^{i k}=\eta^{i k}+\gamma^{i k}+\cdots,
$$

where the $\cdots$ stand for terms that approach zero more rapidly than $\gamma^{i k}$ as $r \rightarrow \infty$.

If we consider the corresponding asymptotic expansion for the $\Gamma_{i k}^{l}$, then we obtain for the first terms (first approximation)

$$
\begin{array}{r}
\Gamma^{l}{ }_{i k}=\frac{1}{2}\left[\eta_{i s} \eta_{k t}\left(\gamma^{s t}-\frac{1}{2} \eta^{s t} \gamma\right)_{, l}-\eta_{k s}\left(\gamma^{s l}-\frac{1}{2} \eta^{s l} \gamma\right)_{, i}\right. \\
\left.-\eta_{i s}\left(\gamma^{l s}-\frac{1}{2} \eta^{l s} \gamma\right)_{, k}\right],
\end{array}
$$

where $\gamma=\eta_{i k} \gamma^{i k}$.

Substituting these expressions in the field equations $R_{i k}=0$, we obtain in the first approximation

$$
\begin{aligned}
R_{\underline{i k}}= & \Gamma^{a}{ }_{i k, a}-\frac{1}{2}\left(\Gamma^{a}{ }_{i a, k}+\Gamma_{a k, i}^{a}\right) \\
= & \frac{1}{2} \eta_{i s} \eta_{k t} \Delta\left(\gamma^{s t}-\frac{1}{2} \eta^{s t} \gamma\right)-\frac{1}{2} \eta_{k s}\left(\gamma^{s a}-\frac{1}{2} \eta^{s a} \gamma\right)_{, i a} \\
& -\frac{1}{2} \eta_{i s}\left(\gamma^{\underline{a s}}-\frac{1}{2} \eta^{a s} \gamma\right)_{, k a}+\frac{1}{2} \gamma, i k=0 \\
& \quad\left(\Delta=\partial^{2} / \partial x_{1}{ }^{2}+\partial^{2} / \partial x_{2}{ }^{2}+\partial^{2} / \partial x_{3}{ }^{2}\right) .
\end{aligned}
$$

We can now normalize our coordinate system so that

$$
\eta_{k s}\left(\gamma \stackrel{s a}{-}-\frac{1}{2} \eta^{s a} \gamma\right)_{a}-\frac{1}{2} \gamma_{, k}=0 .
$$

Then the field equation $R_{\underline{i k}}=0$ in the first approximation becomes

$$
\Delta\left(\gamma^{s t}-\frac{1}{2} \eta^{s t} \gamma\right)=0 .
$$

Considering the fact that the solutions of these equations are to approach zero as $r \rightarrow \infty$ and ignoring terms that approach zero more rapidly than $1 / r$ we obtain

This yields

$$
\gamma^{s t}-\frac{1}{2} \eta^{s t} \gamma=m_{s t} / r \text {. }
$$

$$
\gamma=-\left(m_{11}+m_{22}+m_{33}-m_{44}\right) / r
$$

and the normalization condition (3.9) yields

$$
\begin{aligned}
& -\eta_{k s}\left(m_{s a} x_{a} / r^{3}\right)+\frac{1}{2}\left(m_{11}+m_{22}+m_{33}-m_{44}\right)\left(x_{k} / r^{3}\right)=0, \\
& k=1,2,3 \\
& -\eta_{k s}\left(m_{s a} x_{a} / r^{3}\right)=0, \quad k=4,
\end{aligned}
$$

or

$m_{i k}=\eta_{i k} m$

$m_{4 k}=0 \quad k=1,2,3 ; \quad m=\frac{1}{2}\left(m_{11}+m_{22}+m_{33}-m_{44}\right)$,

hence $m-m_{44}=0$. Equation (3.11) now becomes

$$
\gamma^{s t}-\frac{1}{2} \eta^{s t} \gamma=\eta^{s t} m / r
$$

and from Eq. (3.8) we obtain

$$
\begin{aligned}
\Gamma_{\underline{4 k}}^{l}=\frac{1}{2}\left[-\eta_{4 s} \eta_{k t} m_{s t}\left(x_{l} / r^{3}\right)+\eta_{4 s} m_{s l}\left(x_{k} / r^{3}\right)\right] \\
=\left(m_{44} / 2\right)\left[-\eta_{4 k}\left(x_{l} / r^{3}\right)+\delta_{4}{ }^{l}\left(x_{k} / r^{3}\right)\right] .
\end{aligned}
$$

For the antisymmetric $\gamma^{i k}=\gamma^{i k s}, s$ we obtain

$$
R_{i \underline{v}}=\Gamma_{i \underline{v}, a}^{a}=-\frac{1}{2} \eta_{i s} \eta_{k t} \Delta \gamma^{s t},
$$

and hence the field equations $R_{i k, l}+R_{k l, i}+R_{l i, k}=0$ yield

$$
\Delta \Delta \gamma^{i k l}=0 \text {. }
$$

Here again the condition $\gamma^{i k l} \rightarrow 0$ as $r \rightarrow \infty$ implies $\gamma^{i k l}=0(1 / r)$ and hence $\gamma^{i k}=0\left(1 / r^{2}\right), \quad \Gamma_{i k}^{l}=0\left(1 / r^{2}\right)$, $R_{i k}=0\left(1 / r^{3}\right)$. Thus the terms involving $\mathrm{g}^{i k l}$ in the integrand of (3.7) are $0\left(1 / r^{3}\right)$.

We now choose $C$ in (3.7) to be a two-sphere of radius $R$ in the hyperplane $x_{4}=0$ with the origin as center. We have then

and

$$
n_{i}=x_{i} / r, \quad i=1,2,3,
$$

$$
\begin{aligned}
\int_{C}\left[2 R_{4 k} \mathfrak{g}^{4 k s}-\mathfrak{g}^{4 k} \Gamma^{s}{ }_{4 k}-\mathfrak{g}^{k 4} \Gamma^{s}{ }_{k 4}\right] n_{s} d C \\
=-2 \int_{C} \Gamma^{s}{ }_{44} n_{s} d C+0(1 / R) \\
=-\int_{C} m_{44}\left(x_{s} / r^{3}\right)\left(x_{s} / r\right) d C+0(1 / R) \\
=-\int_{C}\left(m_{44} / r^{2}\right) d C+0(1 / R) \\
=-4 \pi m_{44}+0(1 / R) .
\end{aligned}
$$

If we let $R \rightarrow \infty$, we obtain

$$
m_{44}=0
$$

and according to (3.12),

$$
m=0 \text { and hence } m_{s t}=0, s, t=1,2,3,4 \text {. }
$$

We have thus established the following:

Theorem: If $\mathrm{g}^{i k}$ is a static solution of the field equations which is regular at every point and satisfies the condition $\lim \mathrm{g}^{i k}=\eta^{i k}$ (asymptotic flatness), then we have $\mathfrak{g}^{i k}-\eta^{i k}=o(1 / \boldsymbol{r})$, or in other words the $\mathfrak{g}^{i k}$ represent a mass free field.

\section{THE CENTRALLY SYMMETRIC STATIC FIELD}

In the centrally symmetric static case we can choose our coordinate system so that

$$
\begin{aligned}
& g_{i k}=-A \delta_{i k}+i B \epsilon_{i k s} x_{s}+C x_{i} x_{k} ; \\
& g_{i 4}=D x_{i}+i E x_{i} ; \quad g_{44}=F, 6
\end{aligned}
$$

where $A, B, \cdots, F$ are functions of $r=\frac{1}{2}\left(x_{1}{ }^{2}+x_{2}{ }^{2}+x_{3}{ }^{2}\right)$ and $\epsilon_{123}=1, \epsilon_{i k s}$ is antisymmetric in each pair of indices.

${ }^{6}$ Here and throughout this section the indices have the range $1,2,3$. 
In this coordinate system we have $\mathfrak{g}^{i 4}=H(r) x_{i}$ and the field equation $\mathrm{g}^{i 4}, i=0$ becomes

$$
3 H+2 r H^{\prime}=0 \text {, }
$$

with the solution $H=\mathrm{Cr}^{-\frac{3}{2}}$. Thus if the field is to be regular we must have $g^{i 4}=0$ and hence $g_{i 4}=0$ or $E=0$. It is also well known that we can introduce coordinates so that $g_{\underline{i q}}=0$, that is $D=0$.

We also have $R_{i k}=G(r) \epsilon_{i k s} x_{s}$ so that the field equation $R_{i k, l}+R_{k l, i}+R_{l i, k}=0$ becomes

$$
3 G+2 r G^{\prime}=0
$$

with the solution $G=\mathrm{Cr}^{-\frac{3}{2}}$. Thus if the field is regular we must have $R_{i k}=0$.

For the $\Gamma$ we obtain the following expressions

$$
\begin{aligned}
\Gamma_{i k}^{l}=a \delta_{i k} x_{l}+b\left(\delta_{k l} x_{i}+\delta_{l i} x_{k}\right)+c x_{i} x_{k} x_{l}+i d \epsilon_{k i l} & \\
& +i e \epsilon_{k i s} x_{s} x_{l}+i f\left(\epsilon_{i l s} x_{s} x_{k}+\epsilon_{l k s} x_{s} x_{i}\right) ; \\
\Gamma^{l}{ }_{4 k}= & i \delta_{l k}+i m x_{l} x_{k}+n \epsilon_{l k s} x_{s} ; \quad \Gamma^{4}{ }_{4 k}=p x_{k} ; \quad \Gamma_{44}^{l}=g x_{l},
\end{aligned}
$$

where $a, b, \cdots, q$ are functions of $r$. If we substitute these expressions in the equations $g_{i k ; l}=0$ then we obtain:

$$
\begin{aligned}
& a=\frac{1}{2} \frac{\gamma^{\prime}}{\gamma}-r \frac{C^{\prime}}{\gamma}+\frac{A}{2 \gamma} \frac{\alpha^{\prime}}{\alpha} ; \quad b=\frac{1 \alpha^{\prime}}{4 \alpha} ; \quad c=\frac{C}{2 \gamma}\left(\frac{C^{\prime}}{C}-\frac{\alpha^{\prime}}{\alpha}\right) \\
& d=-\frac{B}{2 A} ; \quad e=\frac{B}{2 \gamma}\left(\frac{C}{A}-\frac{B^{\prime}}{B}+\frac{\alpha^{\prime}}{\alpha}\right) ; f=-\frac{B}{2 A}\left(\frac{B^{\prime}}{B}-\frac{1 \alpha^{\prime}}{2 \alpha}\right) \\
& l=m=n=0 ; \quad p=\frac{1 F^{\prime}}{2 F} ; \quad q=-\frac{1 F^{\prime}}{2} \frac{1}{\gamma},
\end{aligned}
$$

where $\alpha=A^{2}-2 r B^{2}, \gamma=-A+2 r C$. We now consider the field equation

$$
\begin{aligned}
0=R_{44} & =\Gamma^{a}{ }_{44, a}-2 \Gamma^{4}{ }_{4 a} \Gamma^{a}{ }_{44}+\Gamma^{a}{ }_{44} \Gamma^{b}{ }_{a b} \\
& =\left(q x_{a}\right)_{, a}-2\left(p x_{a}\right)\left(q x_{a}\right)+\left(q x_{a}\right)(a+4 b+2 r c) x_{a} \\
& =r q\left[3 / r+2\left(q^{\prime} / q\right)-2\left(F^{\prime} / F\right)+\gamma^{\prime} / \gamma+\alpha^{\prime} / \alpha\right],
\end{aligned}
$$

so we have either $q=0$ which implies $F^{\prime}=0$ and for a suitable time scale $F=1$, or

$$
3 / r+2\left(q^{\prime} / q\right)-2\left(F^{\prime} / F\right)+\gamma^{\prime} / \gamma+\alpha^{\prime} / \alpha=0
$$

and hence

$$
\left(r^{3} q^{2} \gamma \alpha\right) / F^{2}=\text { const.; }
$$

but if the field is to be regular then the determinant $\left|g_{i k}\right|=F \alpha \gamma$ cannot vanish and $\alpha, \gamma, q$ must be regular functions of $r$. This is compatible with the relation $q^{2} \gamma \alpha / F^{2}=$ const. $/ r^{3}$ only if the constant on the right side and $q$ on the left vanish. We have thus established:

We can introduce coordinates so that $g_{i 4}=0, g_{44}=1$, $\Gamma^{l}{ }_{4 k}=\Gamma^{4}{ }_{4 k}=\Gamma^{k}{ }_{44}=0$.

We can now specialize our coordinate system further so that $g_{i k}=-A \delta_{i k}+i B \epsilon_{i k s} x_{s}$ (no $x_{i} x_{k}$ term). This specialization does not introduce singularities, that is if the original field was everywhere regular then the new field is everywhere regular and the asymptotic behavior of the field quantities is preserved. In this system we have $c=0$ and the equations $g_{i k ; l}=0$ become -

$$
\begin{aligned}
\frac{1}{2} A^{\prime} & =A b+B(d+2 r f) \\
\frac{1}{2} B & =-A d \\
\frac{1}{2} B^{\prime} & =-A f+B b \\
0 & =A(a+b)+B(d+2 r f) \\
0 & =A(e+f)+B b .
\end{aligned}
$$

Eliminating $A, B$ and their derivatives from these equations we obtain:

$$
\begin{aligned}
d^{\prime}-f+4 d^{2}(d+2 r f) & =0 \\
a+b-2 d(d+2 r f) & =0 \\
e+f-2 b d & =0 .
\end{aligned}
$$

The remaining field equations are

$$
\begin{aligned}
0=R_{i k}= & 2 r \delta_{i k}\left[a^{\prime}+(a-b / r)+a(a+2 b)\right. \\
& +(1 / r)(d+2 r e)(d+2 r f)]-x_{i} x_{k}\left[(a+2 b)^{\prime}+a^{2}\right. \\
& \left.-2 b^{2}+2(e-f)(d+2 r f)\right]+i \epsilon_{k i s} x_{s}\left[(d+2 r e)^{\prime}\right. \\
& +(2 a-1 / r)(d+2 r f)+(a+2 b+1 / r)(d+2 r e)] .
\end{aligned}
$$

Thus the field quantities $a, \cdots, f$ are to satisfy the equations

$a^{\prime}+(a-b / r)+a(a+2 b)+1 / r(d+2 r e)(d+2 r f)=0$

$b^{\prime}-(a-b / 2 r)-b(a+b)-(1 / 2 r)(d+2 r f)^{2}=0$

$d^{\prime}-f+4 d^{2}(d+2 r f)=0$

$2 r e^{\prime}+f+\left(2 a-4 d^{2}-1 / r\right)(d+2 r f)$

$a+b-2 d(d+2 r f)=0$

$$
+(a+2 b+1 / r)(d+2 r e)=0
$$

$e+f-2 b d=0$.

The last two equations of this system can be used in order to eliminate the functions $a, f$ from the system of equations. From the resulting four differential equations for the functions $b, d, e$ we can eliminate the quantities $b^{\prime}, d^{\prime}, e^{\prime}$ thus obtaining one algebraic equation and three differential equations.

$$
\begin{aligned}
& \left(-1+8 r d^{2}\right)\left[(d-2 r e+4 r b d)^{2}\right. \\
& \left.\quad+(1 / 2 r)(1+2 r b)^{2}-(1 / 2 r)\right]+4 d^{2}=0 \\
& \left(-1+8 r d^{2}\right)(d-2 r e+4 r b d)+2 r d^{\prime}+d=0 \\
& (d-2 r e+4 r b d)^{2}+2 d(1+2 r b)(d-2 r e+4 r b d)-(2 r b)^{\prime}=0 \\
& 2 d(d-2 r e+4 r b d)^{2}+\left(-(3 / 2 r)-3 b+8 r b d^{2}\right) \\
& \quad \times(d-2 r e+4 r b d)+2 r e^{\prime}+(3 d / 2 r)+6 b d+4 r b^{2} d=0 .
\end{aligned}
$$

If we differentiate the first equation then we obtain an equation which is a linear combination of the four equations. Hence one of the three differential equations can be omitted from the system. We omit the last equation and eliminate the quantity $d-2 r e+4 r b d$ from the other equations. We have

$$
d-2 r e+4 r b d=-(1 / 8 d) \frac{\left(-1+8 r d^{2}\right)^{\prime}}{\left(-1+8 r d^{2}\right)},
$$

or if we introduce the abbreviation $\delta=-1+8 r d^{2}$,

$$
d-2 r e+4 r b d=-(1 / 8 d)\left(\delta^{\prime} / \delta\right) \text {. }
$$

The remaining equations then become

$$
\begin{aligned}
& \left(\delta^{\prime} / \delta\right)^{2}=-\left[4(1+\delta) / r^{2}\right]\left(\beta^{2}+1 / \delta\right) \\
& \left(\delta^{\prime} / \delta\right)^{2}=(2 / r) \beta(1+\delta)\left[\left(\delta^{\prime} / \delta+4\left(\beta^{\prime} / \beta\right)\right)\right]
\end{aligned}
$$

with the abbreviation $\beta=1+2 r b$. 
The first equation yields

$$
\begin{aligned}
-4 \beta^{2} & =\left[r^{2} /(1+\delta)\right]\left(\delta^{\prime} / \delta\right)^{2}+4 / \delta \\
2 \frac{\beta^{\prime}}{\beta} & =\frac{\delta^{\prime} / \delta\left[\left(2 r^{2} / 1+\delta\right)\left(\delta^{\prime} / \delta\right)^{\prime}-r^{2} \delta /(1+\delta)^{2}\left(\delta^{\prime} / \delta\right)^{2}+(2 r / 1+\delta)\left(\delta^{\prime} / \delta\right)-(4 / \delta)\right]}{\left(r^{2} / 1+\delta\right)\left(\delta^{\prime} / \delta\right)^{2}+(4 / \delta)} .
\end{aligned}
$$

If we substitute these expressions in the square of both sides of the second equation, then we obtain

$$
\begin{aligned}
\left(\frac{\delta^{\prime}}{\delta}\right)^{4}=-\left[\frac{r^{2}}{1+\delta}\left(\frac{\delta^{\prime}}{\delta}\right)^{2}+\frac{4}{\delta}\right] & \frac{(1+\delta)^{2}}{r^{2}} \\
& \times\left[1+\frac{\left(4 r^{2} / 1+\delta\right)\left(\delta^{\prime} / \delta\right)^{\prime}-2 r^{2} /(1+\delta)^{2}\left(\delta^{\prime} / \delta\right)^{2}+(4 r / 1+\delta)\left(\delta^{\prime} / \delta\right)-(8 / \delta)}{\left(r^{2} / 1+\delta\right)\left(\delta^{\prime} / \delta\right)^{2}+4 / \delta}\right]\left(\delta^{\prime} / \delta\right)^{2} .
\end{aligned}
$$

If we had $\delta^{\prime}=0$, we would have $\delta=-1$ (since $\delta(0)=-1)$ and hence $d=0$ and $B=0$, so that the field would be symmetric. In this case it is known that there exist no non-trivial solutions of the field equations. Thus if we divide both sides of Eq. (4.2) by $\left(\delta^{\prime} / \delta\right)^{2}$ then we obtain

$$
\begin{aligned}
&\left(\frac{\delta^{\prime}}{\delta}\right)^{2}\left[\frac{r^{2}}{1+\delta}\left(\frac{\delta^{\prime}}{\delta}\right)^{2}+\frac{4}{\delta}\right]+\frac{(1+\delta)^{2}}{r^{2}}\left[\frac{4 r^{2}}{1+\delta}\left(\frac{\delta^{\prime}}{\delta}\right)^{\prime}\right. \\
&\left.-\frac{r^{2}(1-\delta)}{(1+\delta)^{2}}\left(\frac{\delta^{\prime}}{\delta}\right)^{2}+\frac{4 r}{1+\delta}\left(\frac{\delta^{\prime}}{\delta}\right)-\frac{4}{\delta}\right]=0 .
\end{aligned}
$$

Now in order to determine the behavior of $\delta$ we remember that according to the result of the preceding section we have $A=1+0\left(r^{-\frac{1}{2}}\right)$ and by an asymptotic expansion we find $B=0\left(r^{-\frac{3}{2}}\right)$. Hence, $d=0\left(r^{-2}\right)$ and $\delta=-1$ at 0 and $\infty$. Since $\delta=-\left(\left|g_{i k}\right| /\left|g_{\underline{i k}}\right|\right) \neq 0$, we have $-1 \leqq \delta<0$, and, since we assumed that $d$ is not identically zero, $\delta$ has a maximum $-1<\delta_{0}<0$ at $r=r_{0}$. From Eq. (4.3) we see that at $r_{0}$ we have

$$
\left(\delta^{\prime} / \delta\right)^{\prime}=\left(1+\delta_{0}\right) / r_{0}^{2} \delta_{0}<0 .
$$

On the other hand we have $\left(\delta^{\prime} / \delta\right)^{\prime}=\delta^{\prime \prime} / \delta_{0}$ and, since $\delta$ is maximal at $r_{0}$, we have $\delta^{\prime \prime} \leqq 0$ there and hence

$$
\left(\delta^{\prime} / \delta\right)^{\prime} \geqq 0 \text {. }
$$

This contradicts Eq. (4.4). Thus we conclude: There exists no static centrally symmetric solution of the field equations which is asymptotically flat and regular throughout.

We remark that this argument remains valid in the case of a real non-symmetric field satisfying the same field equations. In that case the quantities $B, d, e, f, l$, $m$ of the beginning of this section must be considered imaginary (so that the expressions in which they occur become real). The quantity $\delta=-1+8 r d^{2}$ then satisfies $\delta \leqq-1$ with the equality sign for $r=0$ and $r=\infty$. Since we again assume that $d$ is not identically zero $\delta$ must have a minimum $\delta_{0}<-1$ at $r=r_{0}$. At that minimum we have on the one hand

$$
\left(\delta^{\prime} / \delta\right)^{\prime}=\left(1+\delta_{0}\right) / r_{0}^{2} \delta_{0}>0
$$

and on the other hand, since $\delta^{\prime \prime} \geqq 0$,

$$
\left(\delta^{\prime} / \delta\right)^{\prime}=\delta^{\prime \prime} / \delta_{0} \leqq 0 .
$$

